\title{
Producción de zeolitas de baja sílice a partir de caolín colombiano
}

\section{Production of Low-Silica Zeolites from Colombian Kaolin}

\author{
Villaquirán-Caicedo Mónica Alejandra \\ Universidad del Valle, Cali, Colombia \\ Escuela de Ingeniería de Materiales \\ Grupo de Materiales Compuestos, CENM \\ Correo:monica.villaquiran@correounivalle.edu.co
}

\author{
Mejía-De Gutiérrez Ruby \\ Universidad del Valle, Cali, Colombia \\ Escuela de Ingeniería de Materiales \\ Grupo de Materiales Compuestos, CENM \\ Correo: ruby.mejia@correounivalle.edu.co
}

\author{
Gordillo-Suárez Marisol \\ Universidad Autónoma de Occidente, Cali, Colombia \\ Facultad de Ingeniería \\ Correo:mgsuarez44@yahoo.com
}

\author{
Constanza-Gallego Nidia \\ Materials Science \& Technology Division \\ Oak Ridge National Laboratory P.O., EU \\ Correo:gallegonc@gmail.com
}

Información del artículo: recibido: febrero de 2015, reevaluado: marzo de 2015, aceptado: mayo de 2015

\section{Resumen}

En este artículo se presenta un estudio de la producción de zeolita sintética A de baja sílice por medio de la síntesis hidrotermal a partir de un caolín colombiano. La técnica Metodología de Superficie de Respuesta se emplea con el fin de encontrar las condiciones óptimas de proceso (como las relaciones molares $\mathrm{Na}_{2} \mathrm{O} / \mathrm{SiO}_{2}$ y $\mathrm{H}_{2} \mathrm{O} / \mathrm{Al}_{2} \mathrm{O}_{3}$, tiempo y temperatura) que garanticen la calidad de las zeolitas obtenidas. El grado de cristalinidad y la capacidad de intercambio catiónico, se usaron como variables de respuesta. La capacidad de intercambio catiónico se determinó a partir de la Norma NTC 5167. La composición mineralógica y la microestructura de las zeolitas producidas se caracterizaron a través de técnicas como microscopía electrónica de barrido, difracción de rayos $X$ y resonancia magnética nuclear. Los resultados indican que sí es posible la obtención de zeolitas sintéticas a partir del caolín colombiano evaluado. Zeolita tipo A con capacidad de intercambio catiónico $442 \mathrm{cmol} / \mathrm{Kg}$ (valor mayor al obtenido con una zeolita comercial importada empleada en la industria colombiana, $408 \mathrm{cmol} / \mathrm{Kg}$ ) se obtuvo bajo las siguientes condiciones óptimas de procesamiento: relaciones molares $\mathrm{Na}_{2} \mathrm{O} /$ $\mathrm{SiO}_{2}$ de $2.7, \mathrm{H}_{2} \mathrm{O} / \mathrm{Al}_{2} \mathrm{O}_{3}$ de 150 , temperatura de $66^{\circ} \mathrm{C}$ y tiempo de procesamiento de 8 horas.

\section{Descriptores:}

- capacidad de intercambio catiónico

- síntesis hidrotermal

- metacaolín

- zeolita A 


\begin{abstract}
This paper presents a study of the production of a synthetic zeolite of low-silica content by hydrothermal synthesis from Colombian kaolin. The statistical technique Response-Surface Methodology was used to find the optimum process conditions (i.e., molar ratios $\mathrm{Na}_{2} \mathrm{O} / \mathrm{SiO}_{2}$ and $\mathrm{H}_{2} \mathrm{O} / \mathrm{Al}_{2} \mathrm{O}_{3}$, time and temperature) and to ensure the quality of the zeolites obtained. The cation exchange capacity and the degree of crystallinity were used as response variables. The cation exchange capacity was determined following Standard NTC 5167. The mineralogical composition and microstructure of the zeolites produced were characterized through the application of scanning electron microscopy, X-ray diffraction and nuclear magnetic resonance. The results indicate that it is possible to obtain a good quality synthetic zeolite from the Colombian kaolin evaluated. A zeolite type A with a cation exchange capacity of $442 \mathrm{cmol} / \mathrm{Kg}$, a value higher than that exhibited by the same type of zeolite imported for applications in the Colombian industry (i.e., $408 \mathrm{cmol} / \mathrm{Kg}$ ), was obtained with the following processing conditions: molar ratios $\mathrm{Na}_{2} \mathrm{O} / \mathrm{SiO}_{2}$ of 2.7, $\mathrm{H}_{2} \mathrm{O} / \mathrm{Al}_{2} \mathrm{O}_{3}$ of 150 , at temperature $66^{\circ} \mathrm{C}$ and processing time of 8 hours; yielded.
\end{abstract}

\section{Introducción}

Los caolines son rocas constituidas por minerales tipo caolinita, halloysita, nacrita, dickita, acompañados por otros componentes denominados impurezas tales como cuarzo, feldespatos, micas, ferritas, entre otros. Para su utilización, en algunas de sus aplicaciones se requieren tratamientos posteriores para aumentar su pureza, blancura u otras de sus características comerciales importantes (Torres et al., 2011). Específicamente, la caolinita $\left(\mathrm{Al}_{2} \mathrm{Si}_{2} \mathrm{O}_{5}(\mathrm{OH})_{4}\right)$ pertenece a la familia de los filosilicatos y está formada por láminas de tetraedros de sílice y octaedros de alúmina unidos a través de grupos hidroxilo. Este material cuando se somete a altas temperaturas, entre los 600 y $1200^{\circ} \mathrm{C}$, sufre transformaciones estructurales atribuidas a la deshidroxilación (o eliminación de grupos $\mathrm{OH}$ ) de la estructura de la caolinita obteniéndose, aproximadamente a los $700^{\circ} \mathrm{C}$, el metacaolín $\left(\mathrm{Al}_{2} \mathrm{Si}_{2} \mathrm{O}_{7}\right)$. Posteriormente, a temperaturas superiores se obtiene $\gamma-\mathrm{Al}_{2} \mathrm{O}_{3}$ y mullita $\left(3 \mathrm{Al}_{2} \mathrm{O}_{3} \bullet 2 \mathrm{SiO}_{2}\right)$ (Torres et al., 2011; Mejía y Torres, 2003). El metacaolín (MK) es una fase amorfa altamente reactiva y por su gran contenido de aluminosilicatos y su inestabilidad bajo condiciones altamente alcalinas es apto como materia prima para la producción de diversos tipos de zeolitas o feldespatos (Akolekar et al., 1997; San Cristóbal et al., 2010; Chandrasekhar et al., 1997; Miao et al., 2009). La síntesis de zeolitas a partir del MK involucra básicamente dos pasos: la disolución del MK, que conlleva a la formación de un gel aluminosilicato y posteriormente la nucleación y crecimiento de cristales. La presencia de impurezas en el caolín se considera que afecta no solo la reactividad, sino también la calidad de los productos finales. Estudios previos han mostrado que a partir del MK es posible obtener zeolitas de gran calidad y bajo contenido de sílice como son las zeolitas A, $X, Y$. Estas zeolitas se han empleado para la absorción y secado de gases como $\mathrm{CO}_{2}$ y benzeno (Pavlov et al., 2009; Chandrasekhar y Pramada 1999), además de resultar muy útiles para el control de desechos industriales, por ejemplo en la eliminación de contaminantes en aguas residuales como son $\mathrm{Cr}$ (III) $e$ iones de $\mathrm{Cs}$ radioactivo (Covarrubias et al., 2006; Zhao et al., 2004).

Entre los métodos para la síntesis se destacan el método convencional o de síntesis hidrotermal en medio alcalino, el cual se puede realizar en tres etapas (gelificación, envejecimiento y cristalización). La síntesis de zeolitas por el método hidrotermal exige el control de las relaciones $\mathrm{Na}_{2} \mathrm{O} / \mathrm{SiO}_{2}, \mathrm{H}_{2} \mathrm{O} / \mathrm{Al}_{2} \mathrm{O}_{3}$ en la mezcla, así como también la temperatura y el tiempo de síntesis, ya que estas variables influyen en el tipo y calidad de la zeolita formada; también se considera importante la calidad y tipo de materia prima utilizada. Para la síntesis de la zeolita A, de relación $\mathrm{Si} / \mathrm{Al}$ alrededor de 1, es recomendable utilizar temperaturas no mayores a $85^{\circ} \mathrm{C}$. Mientras que para obtener zeolitas $\mathrm{X}$ de relaciones $\mathrm{Si} / \mathrm{Al}$ de hasta 2.5 las temperaturas de síntesis son mayores y además se emplean otros aditivos como fuente de silicatos, pues solo con el caolín, no se alcanza la relación $\mathrm{Si} / \mathrm{Al}$ necesaria para la formación de este tipo de zeolita (Covian, 1991). Se han sintetizado productos de composición $95 \%$ zeolita X y 5\% zeolita A en tiempos de aproximadamente 20h (Akolekar et al., 1997); zeolita $\mathrm{NaX}$ a $24 \mathrm{~h}$ de reacción y $87^{\circ} \mathrm{C}$ para relación molar de $\mathrm{SiO}_{2} / \mathrm{Al}_{2} \mathrm{O}_{3}=3$ y mezclas de fases $\mathrm{NaA}, \mathrm{NaP}$ e hidroxisodalita (HS), termodinámicamente metaestables, a relación $\mathrm{SiO}_{2} / \mathrm{Al}_{2} \mathrm{O}_{3}=7$ (Chandrasekhar y Pramada, 1999). Asimismo se ha producido zeolita Y con adición de sili- 
cato de sodio como fuente adicional de sílice, utilizando un tiempo de síntesis mayor a 4 horas (Chandrasekhar y Pramada, 2004). Se han obtenido Zeolitas de baja sílice (Covarrubias et al., 2006), tales como la zeolita A y la $\mathrm{X}$, por medio del método convencional en una sola etapa con adición de metasilicato de sodio, donde se encontró que después de 5 horas de reacción hidrotérmica se presenta mezcla de fases zeolíticas como faujasita y zeolita A, que cristalizan simultáneamente; sin embargo, luego de tiempos largos de reacción se cristaliza HS $(<240 \mathrm{~h})$ debido a que es una fase termodinámicamente más estable, resultados similares se obtuvieron por otros investigadores (Akolekar et al., 1997). Adicionalmente se reportó la síntesis de zeolita tipo mordenita de altas relaciones $\mathrm{Si} / \mathrm{Al} \sim 20$ y ZSM-5 por medio de la técnica de sembrado (Mignoni et al., 2008) al introducirse fuentes de $\mathrm{Si}$ y Al adicionales al MK. También se ha investigado la síntesis en dos etapas (Miao et al., 2009), la primera de envejecimiento duró un tiempo de $12 \mathrm{~h}$ y posterior cristalización entre 70 y $100^{\circ} \mathrm{C}$.

Pavlov et al. (2009), investigaron la síntesis de zeolita A y $\mathrm{X}$ dejando envejecer los geles a temperatura entre $25-30^{\circ} \mathrm{C}$, reportan que el incremento en el tiempo del envejecimiento de 1 a 24 horas no tiene efecto notorio en la composición de la fase, cristalinidad y características de adsorción de la zeolita; en términos generales después de 8 horas a la temperatura de cristalización empleada $\left(95^{\circ} \mathrm{C}\right)$, los grados de cristalinidad de las zeolitas permanecían prácticamente constantes. Otros métodos para la síntesis de zeolitas son:

1. El ultrasónico (Park et al., 2001), con el cual se reducen los tiempos de síntesis y la temperatura de reacción obteniendo productos como zeolita A con mayor grado de cristalinidad que el logrado por medio del método convencional.

2. El método hidrotermal con previa fusión, en donde el caolín se calcina en soluciones alcalinas de $\mathrm{NaOH}$ o de $\mathrm{KOH}$ alrededor de $\operatorname{los} 600^{\circ} \mathrm{C}$ y posteriormente se continúa con la síntesis convencional, este método se aplica igualmente a otros materiales de partida (Ríos et al.,2006; Ríos et al.,2010a).

3. La síntesis por microondas, que emplea la energía del microondas para la etapa inicial de la síntesis y, a diferencia del método convencional, el gradiente de temperatura en la mezcla se unifica (Chandreskhar y Pramada, 2008).

Las zeolitas sintetizadas a partir de caolín son ligeramente de calidad inferior a las fabricadas con geles de aluminosilicatos de sodio, debido a las impurezas que presenta el caolín (Pavlov et al., 2009) y que pueden afectar las propiedades de la zeolita, tales como la capacidad de intercambio catiónico o su grado de blancura, sin embargo, el empleo del caolín como materia prima reduce mucho los costos de producción de la zeolita sintética comparado al empleo de reactivos químicos como fuentes de silicatos y aluminatos puros. Por ello son necesarios algunos tratamientos previos a las síntesis, para disminuir el contenido de estas impurezas. Para esto existen métodos tanto mecánicos como químicos que mejoran la calidad del caolín. El tratamiento acido a $40^{\circ} \mathrm{C}$ es muy efectivo para la eliminación del Fe del caolín, lo cual permite sintetizar zeolita $\mathrm{NaA}$ apta para el uso en detergentes (Restrepo y Ocampo, 1996). San Cristóbal et al. (2010), compararon el efecto de la activación mecánica y la activación térmica de caolines con diferentes contenidos de cuarzo y caolinita, encontrando que en el proceso de zeolitización las muestras tratadas térmicamente reaccionaron más rápido y que, aunque no se obtiene $100 \%$ de zeolita A, la morfología de los cristales cúbicos es superior.

El objetivo de la presente investigación es evaluar la viabilidad de producción de zeolitas de baja sílice a partir de la síntesis hidrotermal, usando un caolín colombiano tratado térmicamente, y que posee impurezas cuarzo y óxido de hierro. La optimización del proceso se dio por medio de la aplicación de un diseño de experimentos basado en la técnica de metodología de superficie de respuesta (MSR). Este método estadístico conjuga técnicas de diseños experimentales y de optimización y establece la relación entre una o más variables de respuesta y un grupo de factores cuantitativos, además es un método secuencial de experimentación, que presenta como alternativa plantear un experimento único en caso de que la experimentación pueda extenderse a un periodo de tiempo más largo. Para que los tratamientos produzcan sus efectos, la estrategia natural, es tratar de descubrir la combinación óptima al final del experimento único y, en consecuencia, mejorar el rendimiento del proceso mediante el ajuste de variables (Méndez, 1980). En el presente estudio se optimizaron las relaciones molares $\mathrm{Na}_{2} \mathrm{O} / \mathrm{SiO}_{2}, \mathrm{H}_{2} \mathrm{O}$ / $\mathrm{Al}_{2} \mathrm{O}_{3}$, el tiempo y la temperatura; para ello se desarrolló un experimento único empleando la MSR en un diseño completo central en el cual, además del factorial $2^{4}$, se tienen en cuenta otros puntos que son combinaciones de puntos centrados con puntos factoriales, además de los puntos centrados, los cuales serán replicados seis veces. Con base en los resultados logrados se plantea un modelo de predicción del valor de la capacidad de intercambio catiónico en función de las variables de proceso y se determina el grado de cristalinidad de la zeolita producida. 


\section{Materiales y sintesis de las zeolitas}

Materiales

Para el presente estudio se seleccionó un caolín de la región del Valle de Cauca, en Colombia, basado en el análisis de difracción de rayos- $X$, contiene fases de caolinita y cuarzo de 60 y 40\%, respectivamente. La composición química del caolín, determinada por Fluorescencia de Rayos- $X$, la cual se puede apreciar en la tabla 1 , indica la relación molar de los óxidos principales $\mathrm{SiO}_{2} / \mathrm{Al}_{2} \mathrm{O}_{3}$ es de 2.3, ideal para la obtención de zeolitas de baja sílice (Miao et al., 2009; Chandrasekhar y Pramada, 2001).

Tabla 1. Composición química del caolín usado

\begin{tabular}{cc}
\hline Componente & $(\%)$ \\
\hline $\mathrm{SiO}_{2}$ & 46.61 \\
$\mathrm{Al}_{2} \mathrm{O}_{3}$ & 33.69 \\
$\mathrm{TiO}_{2}$ & 1.94 \\
$\mathrm{Fe}_{2} \mathrm{O}_{3}$ & 1.18 \\
$\mathrm{CaO}$ & 0.39 \\
$\mathrm{MgO}$ & 0.21 \\
$\mathrm{~K}_{2} \mathrm{O}$ & 0.04 \\
$\mathrm{~S}$ & 0.80 \\
Pérdida al fuego $\left(1000^{\circ} \mathrm{C}\right)$ & 13.54 \\
\hline
\end{tabular}

\section{Caracterización del MK}

Previo a la síntesis de zeolitas el caolín fue adecuado mecánica y térmicamente para la obtención del MK. El tratamiento mecánico consistió en un proceso de molienda por un tiempo de 25 minutos en un molino de bolas, donde se obtuvo un tamaño de partícula promedio de $11.72 \mu \mathrm{m}$, con $70 \%$ del material de tamaño inferior a $11 \mu \mathrm{m}$. El tratamiento térmico consistió en una quema a $700^{\circ} \mathrm{C}$ por tiempo de una hora en un horno eléctrico, con el fin de liberar el agua estructural y obtener la fase amorfa o MK, aumentando así la reactividad para el proceso de síntesis de las zeolitas (Torres et al., 2011; Mejía y Torres, 2003).

El MK, obtenido a partir del tratamiento térmico del caolín, se caracterizó por difracción de rayos $X$ (DRX), y microscopia electrónica de barrido (MEB). El difractograma logrado del ensayo DRX (figura 1) corrobora la transformación a $\mathrm{MK}$ al desparecer los picos característicos de la caolinita, los cuales se encuentran ubicados en los ángulos $2 \theta$ de $12.14^{\circ}, 19.78^{\circ}, 24.6^{\circ}$ y $34.8^{\circ}$, tal como se reportó en estudios anteriores
(Torres et al., 2011; Mejía y Torres, 2003; Restrepo y Ocampo, 1996; Ríos et al., 2010b). Sin embargo, el difractograma del MK muestra que el material no es totalmente amorfo, pues siguen presentes los picos característicos a cuarzo (Q) ubicados en los ángulos $2 \theta$ de $20.8^{\circ}, 26.6^{\circ}$ y $42.3^{\circ}$. En la misma figura se presenta una imagen MEB de las partículas de caolín luego de la adecuación mecánica en el molino de bolas y en la parte inferior se aprecia la apariencia final después del tratamiento térmico a $700^{\circ} \mathrm{C}$, es decir del MK. En el primer caso se pueden apreciar partículas alargadas laminares y partículas más pequeñas aglomeradas, para el caso del MK (figura 1b) se observan partículas de menor tamaño aglomeradas.

Diseño de experimentos para la síntesis de las zeolitas

Las zeolitas se prepararon a partir del MK utilizando el método de síntesis hidrotermal con una solución de $\mathrm{NaOH}$. Las variables de cada uno de los ensayos se determinaron por el diseño de experimentos, siguiendo la técnica MSR (Méndez, 1980). Para ello, y con ayuda del programa estadístico Minitab 15, se utilizó un diseño completo central, en el cual además del factorial $2^{4}$ se tuvo en cuenta un punto centrado que se replicó seis veces además de las combinaciones de puntos centrados con puntos factoriales, obteniéndose en total 31 experimentos. El código asignado a cada uno de estos fue: NxWyTztm, donde las literales corresponden a $\mathrm{N}$ : $\mathrm{Na}_{2} \mathrm{O} / \mathrm{SiO}_{2}, \mathrm{~W}: \mathrm{H}_{2} \mathrm{O} / \mathrm{Al}_{2} \mathrm{O}_{3}$, T: temperatura y t: tiempo y los subíndices $\mathrm{x}, \mathrm{y}, \mathrm{z}$ y $\mathrm{m}$ pueden tomar valores cada uno de 1, 2 o 3 representando el nivel inferior, centrado y superior para cada factor de estudio, igualmente pue-

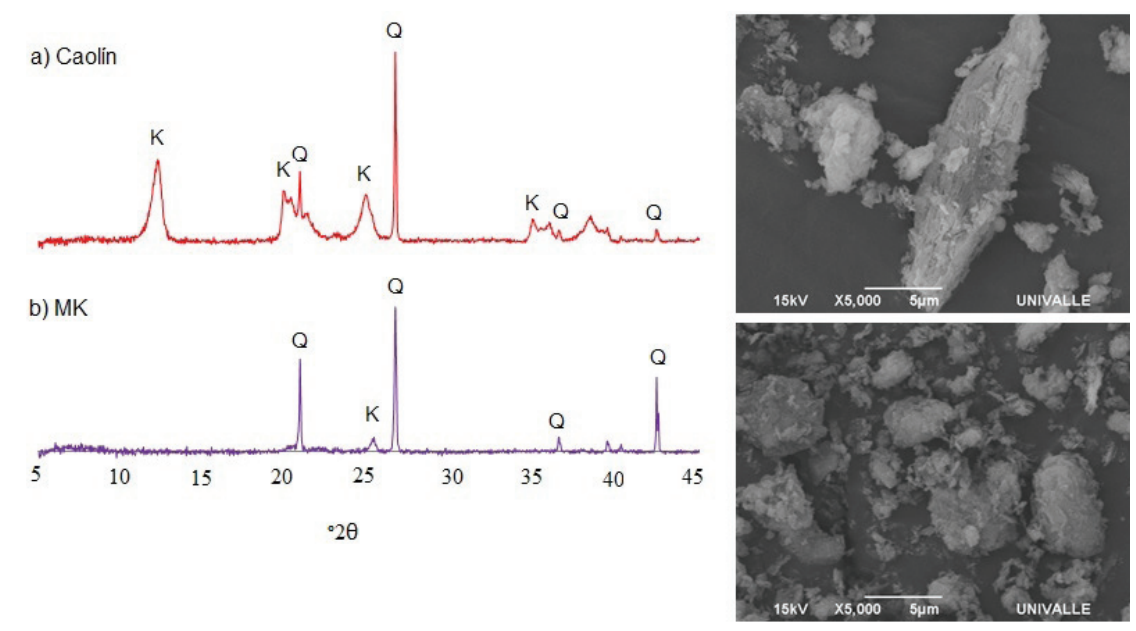

Figura 1. Espectros de DRX e imágenes de MEB para a) caolín y b) metacaolín (K: caolinita, Q: cuarzo) 
den tomar valores de 4 y - 0 cuando corresponden a una mezcla por fuera del diseño (puntos axiales). Las relaciones de este estudio fueron $\mathrm{N}: 1.2,1.7,2.2 ; \mathrm{W}: 75$, 100,$125 ; \mathrm{T}: 65^{\circ} \mathrm{C}, 75^{\circ}, 85^{\circ} \mathrm{C}$; t: $2,4,6$; adicionalmente se escogieron puntos axiales N: 0.7 y 2.7, W: 50 y 150, T: 55 y $95^{\circ} \mathrm{C}$, t: 1 y $6 \mathrm{~h}$. Las variables de respuesta seleccionadas fueron la capacidad de intercambio (CIC) y el grado de cristalinidad (GC).

\section{Caracterización de las zeolitas}

Una vez realizados los procedimientos de síntesis las muestras se filtraron al vacío, posteriormente se lavaron con agua destilada y se secaron a $103^{\circ} \mathrm{C}$ por un periodo de 12 horas, previamente a la determinación de sus características fisicoquímicas y microestructurales. La determinación de la CIC de los productos sintetizados se realizó con acetato de amonio, acorde al procedimiento que se describe en la Norma Colombiana NTC 5167. Las características morfológicas y microestructurales se analizaron a través de diferentes técnicas instrumentales tales como: MEB en un equipo JEOL JSM-6490LV, DRX en un goniómetro de amplio ángulo RINT2000, usando la señal $\mathrm{K}_{\alpha 1}$ del Cu a $45 \mathrm{kV}$ y $40 \mathrm{~mA}$, a un paso de $0.02^{\circ}$ dentro de un rango de $5^{\circ}-40^{\circ}$; resonancia magnética nuclear (RMN) en un equipo BRUKER 400 Ultrashied ADVANCE II 400 utilizando una sonda de sólidos $\left({ }^{27} \mathrm{Al} \mathrm{y}{ }^{29} \mathrm{Si}\right)$. Una vez identificado el tipo de zeolita, y utilizando como referencia una zeolita 4A comercial Standard QB/T 1768-2003, el GC se determinó con base en el difractograma de DRX para cada producto de síntesis siguiendo la norma ASTM D5357.

\section{Resultados y discusión}

Resultados en las muestras preliminares $\mathrm{CIC}$ y $\mathrm{GC}$

La CIC de las zeolitas depende del número de cationes intercambiables, los cuales se determinan por la relación Si/Al (Covarrubias et al., 2006). En la zeolita A, la cual presenta una relación $\mathrm{Si} / \mathrm{Al}$ cercana a 1.0, los iones $\mathrm{Na}^{+}$estabilizan el exceso de carga negativa, que se deja por los átomos de Aluminio presente en la estructura con coordinación tetraédrica. Estos iones de sodio en la zeolita son móviles y pueden intercambiarse con otros cationes en so- luciones acuosas bajo ciertas condiciones. La CIC se determinó a las 31 muestras generadas en el diseño de experimentos con base en la norma, el resultado obtenido para cada experimento se compara con el de las materias primas y una zeolita 4A comercial; adicionalmente se incluye en el gráfico el GC (figura 2). El caolín reporta CIC de $40 \mathrm{cmol} / \mathrm{Kg}$, la activación térmica del caolín disminuye el valor a $14 \mathrm{cmol} / \mathrm{Kg}$, mientras la activación hidrotermal con $\mathrm{NaOH}$ convierte al MK en un material de tipo zeolítico y su CIC se incrementa. Las muestras con geometría cubica típica de zeolita A, evidenciada en los ensayos MEB y DRX, presentaron valores de CIC superiores, incluso la muestra 31-N3W3T3t3 presentó una CIC de $403 \mathrm{cmol} / \mathrm{Kg}$ valor muy cercano al de la zeolita $4 \mathrm{~A}$ comercial $(408 \mathrm{cmol} /$ $\mathrm{Kg}$ ) confirmando la formación de fases zeolíticas.

El GC de las zeolitas se determinó a partir del ensayo de DRX por medio de la ecuación 1, previamente empleada por otros autores (Park et al., 2001), donde I representa la intensidad para la muestra a evaluar y $\mathrm{I}_{0}$ es la intensidad para la muestra de referencia (para este caso la zeolita A comercial) a la cual se le asignó un GC de 100\%. El GC se determinó como la suma de intensidades de los principales picos del difractograma de DRX entre los ángulos $2 \theta=6^{\circ}-40^{\circ}$. Cabe anotar, que un número apreciable de las muestras presentaron altos GC, se destacan las muestras 25-N2W2T2t0 y 31-N3W3T3t3 con GC de 76.31 y $79.52 \%$, respectivamente.

$G C=\left(\frac{I}{I_{0}}\right) * 100$

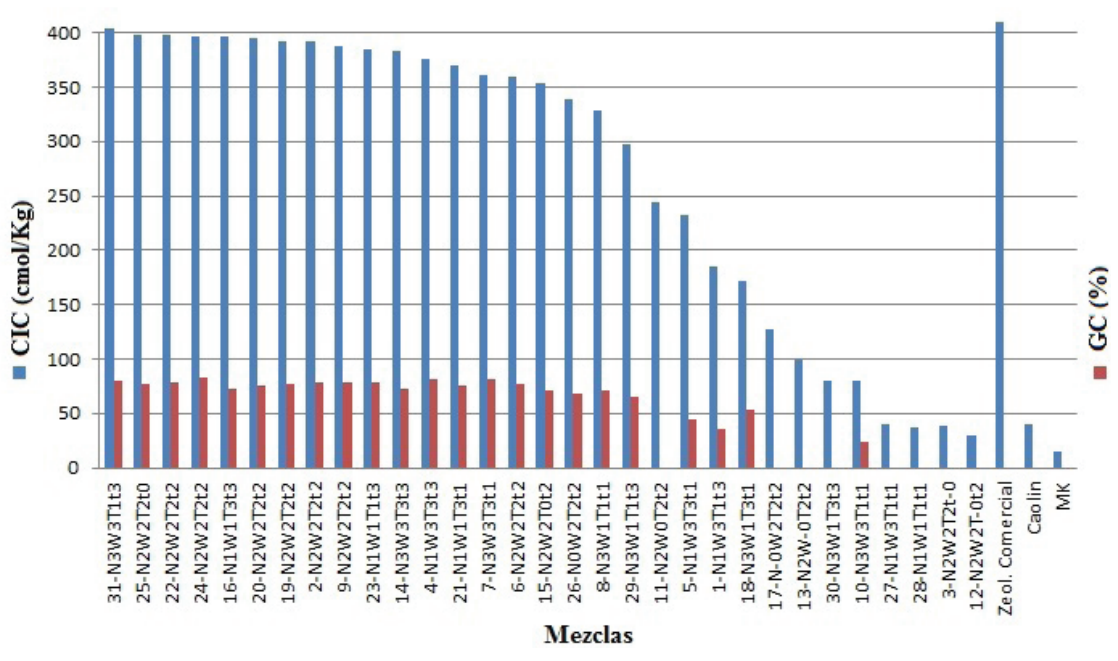

Figura 2. Resultados de la CIC y GC de los productos de síntesis comparados con las materias primas y la zeolita comercial 
Los valores de CIC que se obtuvieron se encuentran en el rango de 79.30 a $403.25 \mathrm{cmol} / \mathrm{Kg}$, el análisis de estos resultados por medio del paquete de análisis estadístico Minitab 15 permitió establecer una relación entre el GC y la CIC, que se expresa con la ecuación 2. Con base en esta ecuación, cuyo $R^{2}$ fue de $94.8 \%$, es posible predecir el GC para las muestras a las que no se les determinó, debido a que en resultados de MEB no mostrados en este artículo no se encontró la morfología cubica típica de la zeolita A; estas son:

3-N2W2T2t-0, 11-N2W0T2t2, 13-N2W-0T2t2, 17-N0W2T2t2, 12-N2W2T-0t2, 27-N1W3T1t1, 28-N1W1T1t1 y $30-N 3 W 1 T 3 t 3$, obteniéndose valores entre $8.64 \%$ para $12-N 2 W 2 T-0 t 2$ y $50.12 \%$ para 11-N2W0T2t2.

$G C=2.83+0.194$ CIC

Por lo tanto, por medio de la CIC es posible predecir el grado de cristalinidad del material, sin hacer uso de la técnica de DRX, por lo cual para objeto de este estudio, solo se optimizó la CIC.

Optimización de la MSR y selección de parámetros de síntesis óptimos para la producción de zeolitas a partir de MK

Con base en el análisis estadístico de los resultados de los 31 ensayos iniciales (figura 2) se definió el modelo general para la CIC presentado en la ecuación 3, cuyo $R^{2}$ fue de $99.3 \%$; en esta ecuación $X_{1}, X_{2}, X_{3}, X_{4}$ corresponden a los factores $\mathrm{Na}_{2} \mathrm{O} / \mathrm{SiO}_{2}, \mathrm{H}_{2} \mathrm{O} / \mathrm{Al}_{2} \mathrm{O}_{3}$, temperatura y tiempo.

$$
\begin{aligned}
& \text { C.I.C }_{i j k l}=-4537.09+676.55 \chi_{1}+-13.82 \chi_{2}+101.41 \chi_{3} \\
& +424.21 \chi_{4}+-93.45 \chi_{1} \chi_{1}+\beta-0.06 \chi_{2} \chi_{2}+-0.52 \chi_{3} \chi_{3} \\
& +-11.26 \chi_{4} \chi_{4}+6.31 \chi_{1} \chi_{2}+-11.22 \chi_{1} \chi_{3}+-31.34 \chi_{1} \chi_{4} \\
& +0.18 \chi_{2} \chi_{3}+0.55 \chi_{2} \chi_{4}+-3.88 \chi_{3} \chi_{4}+\varepsilon_{i j k l}
\end{aligned}
$$

En el comportamiento experimental, se evidencia que si la CIC dependiera solo de las relaciones molares $\left(\mathrm{Na}_{2} \mathrm{O} /\right.$ $\mathrm{SiO}_{2}$ y H $\mathrm{H}_{2} \mathrm{O} / \mathrm{Al}_{2} \mathrm{O}_{3}$ ) estas serían directamente proporcionales a CIC, tal como se aprecia en los gráficos de contorno (figura 3a). Sin embargo, al entrar otras variables al sistema como temperatura y tiempo, la CIC se favorece por bajas relaciones molares $\left(\mathrm{Na}_{2} \mathrm{O} / \mathrm{SiO}_{2}\right)$ a altas temperaturas y tiempo de síntesis (figura $3 \mathrm{~b}$ y $3 \mathrm{c}$ ). Para el caso del segundo factor (relación molar $\mathrm{H}_{2} \mathrm{O} / \mathrm{SiO}_{2}$ ), se aprecia que este tiene un efecto significativo para la CIC, logrando una maximización de la CIC cuando la temperatura y el tiempo aumentan, sin embargo cuando la temperatura sobrepasa los $90^{\circ} \mathrm{C}$ se aprecia un decrecimiento de CIC. El efecto de la temperatura y el tiempo en CIC (figura 3f), el tiempo y la temperatura actúan de forma inversa, así temperaturas por debajo de $75^{\circ} \mathrm{C}$ requieren tiempos de formación del gel superiores; similares resultados se encontraron por Covian (1991). Los valores óptimos de CIC (entre $400 \mathrm{cmol} / \mathrm{Kg}$ y $500 \mathrm{cmol} / \mathrm{Kg}$ ) se obtienen a las siguientes condiciones: relación molar $\mathrm{Na}_{2} \mathrm{O} / \mathrm{SiO}_{2}$ entre 2.3 y 2.5, relación molar $\mathrm{H}_{2} \mathrm{O} / \mathrm{SiO}_{2}$ entre 120 y 140 , temperatura de 85 a $95^{\circ} \mathrm{C}$ y tiempo entre 5 y $8 \mathrm{~h}$.
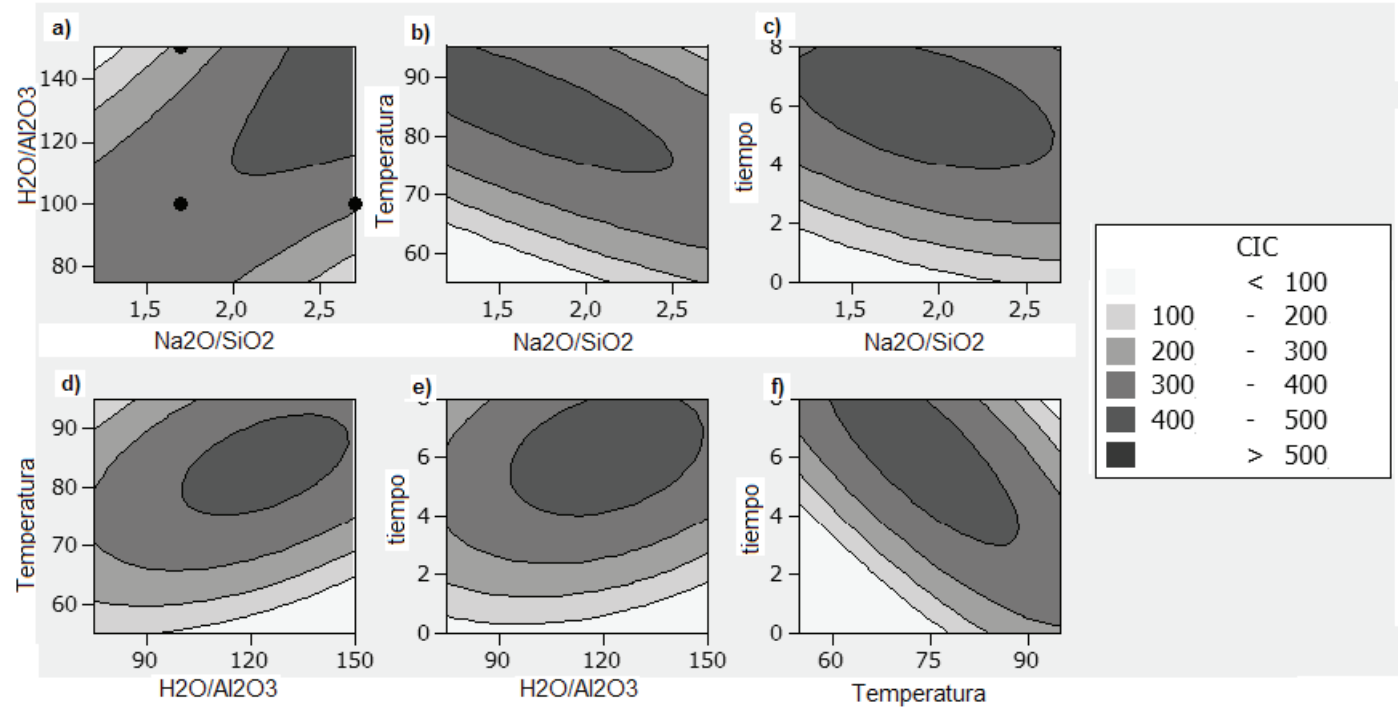

Figura 3. Gráficos de contorno para la CIC de los productos de síntesis a partir de MK 
Síntesis y caracterización bajo condiciones óptimas

A partir de la aplicación de la MSR se generaron tres posibles optimizaciones de las variables de síntesis de zeolita. Estas condiciones se aplicaron a nuestro MK y se obtuvieron los productos OPT1, OPT2 and OPT3. Adicionalmente, los mismos parámetros de síntesis de óptimos se aplicaron a un MK de origen comercial de alta pureza importado $\left(\right.$ Metamax $\left.^{\mathrm{TM}}\right)$, y se obtuvieron los productos OPT1*, OPT2* y OPT3*, que serán utilizados como punto de comparación con las mismas condiciones de procesamiento (tabla 2).

Los resultados de la CIC de los experimentos bajo condiciones óptimas se muestran en la tabla 3. Se aprecia que al incrementar la pureza del MK es posible obtener valores superiores a los que predice el modelo hasta $15 \%$ y con GC de $100 \%$; se incluyen microfotografías por MEB y el espectro de DRX (figura 4) para comparar las muestras OPT1 y OPT1* (las cuales presentaron la mayor CIC) con la zeolita $4 \mathrm{~A}$ comercial. En estas imágenes (figura 4) se puede apreciar la morfología cúbica característica de la zeolita A. En la gran mayoría de los productos los cubos obtenidos presentaban esquinas rectas, pero hubo excepciones (resultado no mostrado en este artículo) donde los cubos eran de es-

Tabla 2. Condiciones de procesamiento para la síntesis en condiciones óptimas

\begin{tabular}{|c|c|c|c|c|}
\hline \multirow[b]{2}{*}{ Óptimos } & \multicolumn{2}{|c|}{ Relación molar } & \multirow{2}{*}{$\begin{array}{l}\mathrm{T} \\
{ }^{\circ} \mathrm{C}\end{array}$} & \multirow{2}{*}{$\begin{array}{l}\mathrm{t} \\
\mathrm{h}\end{array}$} \\
\hline & $\mathrm{Na}_{2} \mathrm{O} / \mathrm{SiO}_{2}$ & $\mathrm{H}_{2} \mathrm{O} / \mathrm{Al}_{2} \mathrm{O}_{3}$ & & \\
\hline $\begin{array}{l}\text { OPT1/ } \\
\text { OPT1* }\end{array}$ & 2.7 & 150 & 66 & 8.0 \\
\hline $\begin{array}{l}\text { OPT2/ } \\
\text { OPT2* }\end{array}$ & 2.0 & 116 & 72 & 6.44 \\
\hline $\begin{array}{l}\text { OPT3/ } \\
\text { OPT3* }\end{array}$ & 1.2 & 75 & 76 & 5.46 \\
\hline
\end{tabular}

Tabla 3. CIC y GC bajo condiciones óptimas

\begin{tabular}{ccccc}
\hline & \multicolumn{2}{c}{ CIC $(\mathrm{cmol} / \mathrm{Kg})$} & $\begin{array}{c}\text { Variación } \\
\text { CIC, } \%\end{array}$ & GC, $\%$ \\
\cline { 2 - 4 } Óptimos & Predicha & Experimental & & \\
\hline OPT1 & 503 & 442 & -12.12 & 88.57 \\
OPT2 & 444 & 422 & -4.95 & 84.70 \\
OPT3 & 441 & 397 & -9.97 & 79.85 \\
OPT1* & 503 & 506 & +0.59 & 100 \\
OPT2 $^{*}$ & 444 & 499 & +12.38 & 99.60 \\
OPT3* & 441 & 507 & +14.96 & 100 \\
Caolín & - & 40 & - & - \\
MK & - & 14 & - & - \\
Zeolita 4A & - & 408 & - & 100 \\
Comercial & - & & & \\
\hline
\end{tabular}

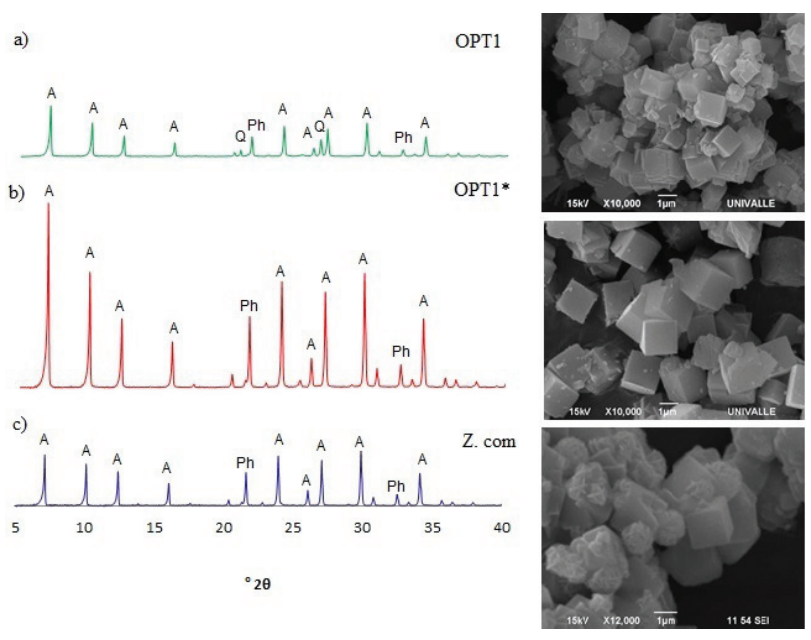

Figura 4. Espectros de DRX y MEB de las zeolitas obtenidas en los tratamientos: a) OPT1, b) OPT1* comparados con la c) zeolita 4A comercial (A: zeolita A, Ph: philipsita, Q: quarzo)

quinas redondeadas, similares a los logrados por Chunfeng et al. (2009).

En general, el tamaño de los cubos fue mayor a los obtenidos con el MK comercial bajo las mismas condiciones de síntesis; para el producto OPT1* fue entre 1.41.6 $\mu \mathrm{m}$ mientras que para el producto OPT1 fueron menores a $1 \mu \mathrm{m}$. También puede apreciarse que en la zeolita 4A comercial se presentan bolas tipo "bola de algodón" distinguibles de la zeolita PHI o Philipsita (Ríos y William, 2008). En general, los productos cristalinos alcanzados se identificaron como zeolita A (figura 4) identificable por los ángulos de difracción $2 \theta$ de $10.19^{\circ}, 12.49^{\circ}, 16.14^{\circ}, 21.02^{\circ}, 27.18^{\circ}$ y $30.01^{\circ}$, con impurezas en pequeña cantidad de zeolita PHI, la cual se identifica para los ángulos $2 \theta$ de $21.51^{\circ}$ y $32.44^{\circ}$. Adicionalmente se aprecia la presencia de cuarzo (identificable a ángulos $2 \theta$ de $20.86^{\circ}$ y $26.6^{\circ}$ ) proveniente del MK. Cabe notar que la presencia de cuarzo en el producto final no afecta las propiedades de intercambio de la zeolita, pues los valores ganados son satisfactorios y mayores que el CIC de la zeolita $4 \mathrm{~A}$ comercial. La cristalinidad de la zeolita OPT1 fue de $88.57 \%$ y para el

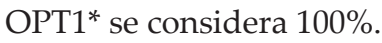

Los aluminosilicatos como las arcillas y las zeolitas contienen unidades de $\mathrm{SiO}_{4}$ y $\mathrm{AlO}_{6} / \mathrm{AlO}_{4}$, las cuales pueden estudiarse por espectroscopía de RMN en estado sólido mediante la sonda de ${ }^{29} \mathrm{Si} \mathrm{y}{ }^{27} \mathrm{Al}$. La sonda de silicio brinda información sobre el tipo de silicato formado $Q^{1}, Q^{2}, Q^{3}$ o $Q^{4}$, como también la sustitución de este en la estructura por otra especie, así la nomenclatura $\mathrm{Q} n(m \mathrm{Al})$ indica que $n$ es el número de átomos de oxigeno que se está compartiendo con otro silicato o aluminato tetraédrico y $m$ representa el número de gru- 

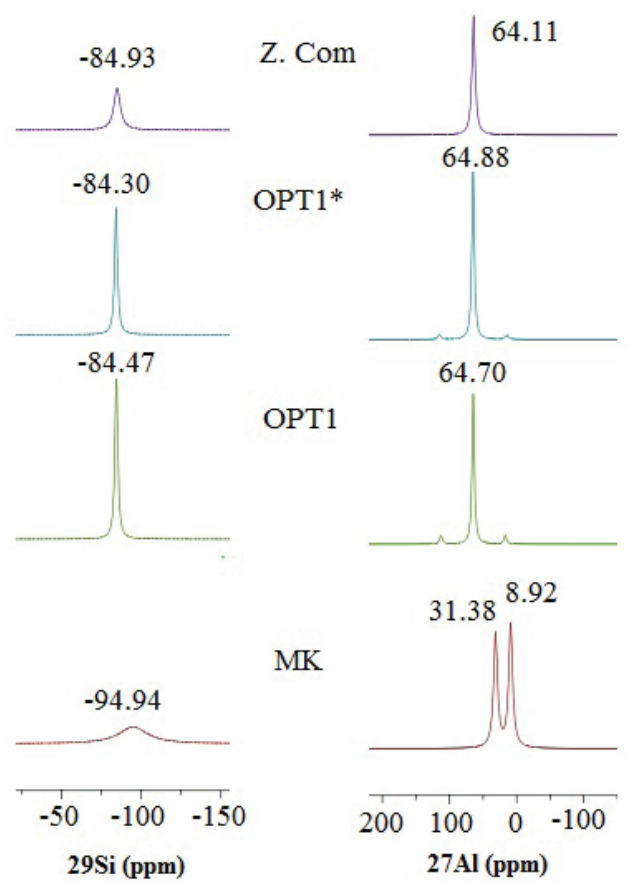

Figura 5. Espectros de RMN 29Si y 27Al de los productos OPT1 y OPT1* comparados con el MK y la zeolita 4A comercial

pos aluminatos vecinos (Scheneider et al., 2001), la señal para el $\mathrm{Si}^{\mathrm{IV}}$ se ubica entre $-70 \mathrm{a}-110 \mathrm{ppm}$, pero su posición exacta depende de los posibles cationes del entorno. Cuando se utiliza la sonda de Aluminio se observan señales ubicadas acorde a la coordinación de este, ya sea tetraédrica $(+50 \mathrm{a}+70 \mathrm{ppm})$, pentaédrica (+30 ppm) u octaédrica $(-10 \mathrm{a}+10 \mathrm{ppm})$. Los espectros RMN obtenidos (figura 5) para el MK de partida, algunos de los productos de síntesis y una zeolita $4^{\mathrm{a}}$ comercial. Se puede observar para el MK señales en + 8.9 ppm $\left(\mathrm{Al}^{\mathrm{VI}}\right)$ y en +31.38 ppm $\left(\mathrm{Al}^{\mathrm{V}}\right)$ ppm, correspondientes a la coordinación hexa y penta del aluminio en la estructura (Chandrasekhar y Pramada, 1999). Posterior al proceso de síntesis se observa en los productos un desplazamiento de la señal hacia la izquierda ubicándose alrededor de $+64 \mathrm{ppm}$, en forma similar a lo observado en la zeolita $4 \mathrm{~A}$ comercial; se confirma así la presencia mayoritaria de $\mathrm{Al}^{\mathrm{IV}}$ en el producto final. En cuanto al espectro alcanzado con la sonda ${ }^{29} \mathrm{Si}$ para el MK se aprecia una señal ancha alrededor de $-94.9 \mathrm{ppm}$ correspondiente a $\mathrm{Q}^{4}(0 \mathrm{Al})$ atribuible a polimorfismos de sílice (Thompson y Barron, 1987) y presencia de sílice amorfa (Rocha y Klinowski, 1990). Con el tratamiento hidrotermal la señal se desplaza a valores hacia la izquierda, la estructura de la zeolita A está formada por uniones de sodalita a través de anillos dobles de cuatro miembros que presentan se- ñales características para los desplazamientos alrededor 83,84 y 85 ppm, indicando la transformación y máximo grado de sustitución isomorfa dando como resultado la formación de enlaces $\mathrm{Q}^{4}(4 \mathrm{Al})$ característicos de la zeolita A (Rios et al., 2010b; Chandrasekhar y Pramada, 2008).

\section{Conclusiones}

Se demostró que es posible sintetizar zeolitas tipo A de buena calidad comparable a las disponibles comercialmente, usando como materia prima caolines colombianos. Las zeolitas se produjeron por tratamiento hidrotermal de un MK, adquirido por tratamiento mecánicotérmico de un caolín colombiano con altos contenidos de cuarzo $(40 \%)$. Las zeolitas obtenidas fueron tipo A, las cuales exhibieron altas CIC de hasta $442 \mathrm{cmol} / \mathrm{Kg}$; mostraron un GC superior a $85 \%$ y un tamaño promedio de partícula de 1 micra. Las condiciones óptimas de procesamiento fueron: relaciones molares $\mathrm{H}_{2} \mathrm{O} / \mathrm{Al}_{2} \mathrm{O}_{3} \mathrm{y}$ $\mathrm{Na}_{2} \mathrm{O} / \mathrm{SiO}_{2}$ de 2.7 y 150 , respectivamente, temperatura de $66^{\circ} \mathrm{C}$, y un tiempo de procesamiento de 8 horas. Cuando estos parámetros de síntesis se aplicaron a un MK comercial de elevada pureza la CIC de la zeolita producida fue ligeramente mayor $(507 \mathrm{cmol} / \mathrm{Kg})$. Los resultados en este estudio muestran que es posible la obtención de zeolitas de calidad comparables a las disponibles comercialmente, abriendo una importante perspectiva a la producción industrial de un material, el cual es de alto costo, se importa y es de alta demanda en el sector industrial colombiano.

\section{Agradecimientos}

Los autores agradecen a los patrocinadores de este estudio: Universidad del Valle (Colombia), el Centro de Excelencia de Nuevos Materiales (CENM) y al Departamento Administrativo de Ciencia, Tecnología e Innovación, Colciencias. La participación de N.C. Gallego fue financiada por una subvención de la beca Fulbright Colombia Commission.

\section{Referencias}

Akolekar D., Chafee A., Howe R.F. The transformation of kaolin to low-silica X zeolite. Zeolites, volumen 19, 1997: 359-265.

Chandrasekhar S., Raghavan P., Sebastian G., Damodaran A.D. Brightness improvement studies on kaolin based zeolite 4A. Applied Clay Science, volumen 12, 1997: 221-231.

Chandrasekhar S. y Pramada P.N. Investigation on the synthesis of zeolite $\mathrm{NaX}$ from kerala kaolin. Journal of Porous Materials, volumen 6, 1999: 283-297. 
Chandrasekhar S. y Pramada P.N. Sintering behavior of calcium exchanged low silica zeolites synthesized from kaolin. Ceramics International, volumen 27, 2001: 105-114.

Chandrasekhar S. y Pramada P.N. Kaolin based zeolite Y, a precursor for cordierites ceramics. Applied Clay Science, volumen 27, 2004: 187-198.

Chandrasekhar S. y Pramada P.N. Microwave assisted synthesis of zeolite A from metakaolin. Microporous and Mesoporous Materials, volumen 108, 2008: 152-161.

Chunfeng W., Jiansheng Li., Xia S., Lianjun W., Xiuyun S. Evaluation of zeolites synthesized from fly ash as potential adsorbents for wastewater containing heavy metals. Journal of Environmental Sciences, volumen 21, 2009: 127-36.

Covarrubias C., García R., Arriagada R., Yánez J., Garland M.T. $\mathrm{Cr}(\mathrm{III})$ exchange on zeolites obtained from kaolin and natural mordenite. Microporous and Mesoporous Materials, volumen 88, 2006: 220-231.

Covian-Sanchez I. Sintesis de zeolitas $13 X$ para uso en detergentes, tesis (doctorado en ciencias químicas), España, Universidad Complutense de Madrid, Facultad de Ciencias Químicas, 1991, 403 p.

Mejía- De Gutiérrez R. y Torres J. Puzolana obtenida por activación térmica, en: Memorias III Jornadas Iberoamericanas de materiales de construcción, Red Iberoamericana de Rocas y Minerales Industriales (XIII-C-CYTED), San Juan, Argentina, 2003, p. 25-29.

Méndez I. Metodología de superficie de respuesta, (Mendez I editor), Instituto de investigaciones en matemáticas aplicadas y en sistemas, UNAM, México, 1980, pp. 15-17.

Miao Q., Zhou Z., Yang J., Lu J., Yan S., Wang J. Synthesis of NaA zeolite from kaolin source. Frontiers of Chemical Engineering China, volumen 3 (número 1), 2009: 8-11.

Mignoni M., Petkowicz D.I., Fernandes N. Sibele B.C. Synthesis of mordenite using kaolin as $\mathrm{Si}$ and $\mathrm{Al}$ source. Applied Clay Science, volumen 41, 2008: 99-104.

Park J., Chan B., Soo S., Chan H. Conventional versus ultrasonic synthesis of zeolite 4A from kaolin. Journal of Materials Science Letters, volumen 20, 2001: 531-533.

Pavlov M.L., Travkina O.S., Basimova R.A., Pavlova I.N., Kutepov B.I. Binder-free synthesis of high-performance zeolites A and X from kaolin. Petroleum, volumen 49 (número 1), 2009: 36-41.
Restrepo G.M. y Ocampo G.A. Sustitución de polifosfatos por zeolitas en detergentes. Revista Facultad Ingeniería Química Universidad de Antioquia, volumen 13, 1996: 15-20.

Ríos C.A., Williams C.D., Castellanos O.M. Síntesis y caracterización de zeolitas a partir de la activación alcalina de caolinita y subproductos industriales (cenizas volantes y clincker natural) en soluciones alcalina. BISTUA, volumen 4 (número 2), 2006: 60-71.

Ríos C.A. y Williams C.D. Synthesis of zeolitic materials from natural clinker: A new alternative for recycling coal combustion by-products. Fuel, volumen 87, 2008: 2482-2492.

Ríos C.A. y Denver C.W. Hydrothermal transformation of kaolinite in the system $\mathrm{K}_{2} \mathrm{O}-\mathrm{SiO}_{2}-\mathrm{Al}_{2} \mathrm{O}_{3}-\mathrm{H}_{2} \mathrm{O}$. DYNA, volumen 77 (número 163), 2010a: 55-63.

Ríos C.A. y Denver C.W., Castellanos OM. Synthesis of zeolite LTA from thermally treated kaolinite. Revista Facultad de Ingeniería Universidad de Antioquia, volumen 53, 2010b: 30-41.

Rocha J. y Klinowski J. ${ }^{29} \mathrm{Si}$ and ${ }^{27} \mathrm{Al}$ magic-angle-spining NMR studies of the thermal transformation of kaolinite. Journal of Physics and Chemistry of Minerals, volumen 17, 1990: 179-186.

San Cristóbal A.G., Castelló R., Luengo M.A., Vizcayno C. Zeolites prepared from calcined and mechanically modified kaolins a comparative study. Applied Clay Science, volumen 49, 2010: 239-246.

Schneider J., Cincotto M.A., Panepucci H. ${ }^{29} \mathrm{Si}$ and ${ }^{27} \mathrm{Al}$ high-resolution NMR characterization of calcium silicate hydrate phases in activated blast-furnace slag pastes. Cement and Concrete Research, volumen 31 (número 7), 2001: 993-1001.

Thompson J.G., Barron P.F. Further consideration of the ${ }^{29} \mathrm{Si}$ nuclear Magnetic Resonance Spectrum of kaolinite. Clays and Clay Minerals, volumen 35, 1987: 38-42.

Torres J., Mejía-De Gutiérrez R., Castelló R., Vizcayno C. Análisis comparativo de caolines de diferentes fuentes para la producción de metacaolín. Revista Latinoamericana de Metalurgia y Materiales, volumen 31 (número 1), 2011: 35-43.

Zhao H., Deng Y., Harsh J.B., Markus F., Boyle J.S. Alteration of kaolinite to cancrinite and sodalite by simulated Hanford tank waste its impact on cesium retention. Clays and Clay Minerals, volumen 52, 2004:1-13. 


\section{Este artículo se cita:}

\section{Citación estilo Chicago}

Villaquirán-Caicedo, Mónica Alejandra, Ruby Mejía-De Gutiérrez, Marisol Gordillo-Suárez, Nidia Constanza Gallego. Producción de zeolitas de baja sílice a partir de caolín colombiano. Ingeniería Investigación y Tecnología, XVII, 01 (2016): 109-118.

\section{Citación estilo ISO 690}

Villaquirán-Caicedo M.A., Mejía-De Gutiérrez R., Gordillo-Suárez M., Gallego N.C. Producción de zeolitas de baja sílice a partir de caolín colombiano. Ingeniería Investigación y Tecnología, volumen XVII (número 1), enero-marzo 2016: 109-118.

\section{Semblanzas de los autores}

Mónica Alejandra Villaquirán-Caicedo. Ingeniera de materiales de la Universidad del Valle (Cali, Colombia) y magister en ingeniería por la misma institución. Es miembro activo del grupo de investigación de materiales compuestos, categoría A1-2015 de Colciencias. Se ha ligado como joven investigador y asistente de investigaciones, ha participado en varios proyectos en la línea de investigación: utilización de desperdicios industriales. Actualmente es candidata al doctorado en ingeniería, estudios que se financian por medio de la beca otorgada por Colciencias.

Ruby Mejía-De Gutiérrez. Química por la Universidad del Valle con master en química por la misma institución. PhD en ciencias químicas por la Universidad Complutense de Madrid. Es directora del grupo de materiales compuestos desde su formación (1986). Ha sido directora de la Escuela de Ingeniería de Materiales de la Universidad del Valle durante el período 2000-2006. Actualmente es profesora en la Universidad del Valle y Coordinadora del programa de Posgrado de la Escuela de Ingeniería de Materiales.

Marisol Gordillo-Suárez. Profesional en estadística, con master en ingeniería de sistemas y PhD en ingeniería de materiales por la Universidad del Valle. Cuenta con una extensa experiencia en el diseño de experimentos y series de tiempo aplicadas a la ingeniería. Actualmente es profesora en la Universidad Autónoma de Occidente (Cali) e investigadora en el Grupo de Materiales Compuestos.

Nidia Constanza-Gallego. Ingeniera industrial de la Universidad del Valle, PhD in Materials Science and Engineering por la Universidad de Clemson (EU). Es investigadora en Oak Ridge National Laboratory (Tennessee, Estados Unidos). 